\title{
USO DE NANOBASTÕES DE OURO PARA O DESENVOLVIMENTO DE AULAS PRÁTICAS DE NANOTECNOLOGIA
}

\author{
Cyntia Silva Ferreira ${ }^{a, *}$, Luiz Orlando Ladeira ${ }^{\mathrm{b}}$, Cristiano Fantini Leite, Flávio Guimarães da Fonseca ${ }^{\mathrm{c}}$, Erica Milena \\ de Castro Ribeiroa, Alice Freitas Versianic, Jorge Fernando de Souza Silva ${ }^{a}$, Cíntia Lopes de Brito Magalhães ${ }^{a}$ e Breno de \\ Mello Silva ${ }^{a}$ \\ aDepartamento de Ciências Biológicas, Universidade Federal de Ouro Preto, 35400-000 Ouro Preto - MG, Brasil \\ bDepartamento de Física, Universidade Federal de Minas Gerais, 31270-901 Belo Horizonte - MG, Brasil \\ 'Departamento de Microbiologia, Instituto de Ciências Biológicas, Universidade Federal de Minas Gerais, 31270-901 Belo \\ Horizonte - MG, Brasil
}

Recebido em 05/10/2016; aceito em 09/01/2017; publicado na web em 14/02/2017

\begin{abstract}
USE OF GOLD NANORODS FOR THE DEVELOPMENT OF PRACTICAL CLASSES IN NANOTECHNOLOGY. A gold nanoparticles functionalization experiment was conducted in a biotechnology summer course at UFOP as a model for the introduction of the laboratory practice in nanotechnology for postgraduate courses in the areas of chemistry and biotechnology. The gold nanorods were synthesized by the seed method and then, functionalized with anti-IL-6 antibodies using the reagents EDAC/NHS and polyethyleneimine (PEI). This nanocompound was tested against the binding with the specific antigen (IL-6) and changes in the longitudinal plasmon absorption spectrum showed the coupling efficiency, which was also verified by the decrease in zeta potential. The experiment was satisfactory, with a positive feedback from participants, and could be implemented in nanotechnology practical classes from postgraduate courses, as a way for improve education in the emergent area of nanobiotechnology.
\end{abstract}

Keywords: gold nanorods; surface plasmon resonance; nanotechnology experiment.

\section{INTRODUÇÃO}

O universo da nanotecnologia se expandiu consideravelmente na esfera da pesquisa científica em todo mundo nos últimos anos e constitui uma área que certamente acarretará em enormes retornos econômicos. Materiais e tecnologias em escala nanométrica têm sido empregados em diversas áreas, tais como no setor alimentício, sistemas de energia renovável, bioinformática e na medicina. ${ }^{1-4}$ Além disso, constituem matéria-prima nobre para o desenvolvimento de dispositivos e sistemas multifuncionais que se adequam aos mais variados produtos e processos industriais.

Perante a complexidade e relevância da nanotecnologia, emerge a necessidade de abordagens práticas e sistêmicas nos cursos de graduação e também de pós-graduação. Entretanto, apesar de já desenvolvidos e utilizados há algum tempo em pesquisas de diversas áreas, os conceitos da nanotecnologia ainda não são abordados em muitos cursos teóricos e práticos de pós-graduação no Brasil.

Neste contexto, uma classe de nanomateriais de grande potencial tecnológico e educacional são as nanopartículas de ouro (AuNPs), que possuem propriedades ópticas, magnéticas e estruturais interessantes para aplicações químicas e biológicas. São ainda as nanopartículas metálicas mais estáveis que se tem conhecimento. ${ }^{5,6}$ Elas podem ser sintetizadas em diversos tamanhos e formatos, tais como nanoesferas, nanobastões, nanogaiolas, nanoestrelas e nanoconchas. ${ }^{5} \mathrm{~A}$ síntese das AuNPs geralmente é econômica, pois embora demande reagentes onerosos, estes são empregados em concentrações muito baixas. ${ }^{7}$

Entre as várias aplicações das nanopartículas de ouro, o sensoriamento é tido como uma das áreas de maior interesse na atualidade. Nesse contexto, dentre as diferentes formas de nanopartículas, é dada grande atenção às nanoesferas e nanobastões de ouro. Isso se deve ao grande volume de conhecimento obtido sobre a característica mais peculiar das nanopartículas de ouro, a chamada ressonância plasmônica

*e-mail: csf.ferreira@gmail.com de superfície (SPR). A SPR se caracteriza pela oscilação coletiva dos elétrons de condução das partículas metálicas desencadeada pela exposição à luz, a qual pode ser detectada por espectroscopia. ${ }^{8,9}$

Descritas pela primeira vez por Faraday em 1857, as nanoesferas de ouro são usualmente sintetizadas a partir de uma reação de redução aquosa de ácido cloroáurico $\left(\mathrm{HAuCl}_{4}\right)$ por citrato de sódio. ${ }^{10,11} \mathrm{Já}$ os nanobastões de ouro são frequentemente sintetizados pelo método mediado por sementes, descrito por Murphy e colaboradores em $2001,12,13$ no qual os nanobastões são preparados à partir da adição de nanoesferas recobertas por citrato (sementes), à uma solução contendo $\mathrm{HAuCl}_{2}$ em elevadas concentrações, resultante da redução de $\mathrm{HAuCl}_{4}$ por ácido ascórbico $\left(\mathrm{C}_{6} \mathrm{H}_{8} \mathrm{O}_{6}\right)$ na presença do surfactante brometo de cetiltrimetilamônio (CTAB) e íons de prata. O CTAB é essencial para a síntese controlada em forma de bastões, devido ao fenômeno de ligação preferencial às diferentes faces dos bastões após o início do crescimento das sementes. ${ }^{14}$

Quanto à propriedade de SPR e sua aplicabilidade como sensores, as nanoesferas de ouro apresentam uma banda de absorção plasmônica na região do espectro visível, em torno de $520 \mathrm{~nm}$. Por outro lado, os nanobastões exibem duas bandas de SPR, visto que a oscilação coletiva dos elétrons pode ocorrer em dois sentidos, dependendo da polarização da luz incidente. Assim, para os nanobastões existe uma banda de absorção no comprimento de onda semelhante ao das nanoesferas (denominada banda transversal), ${ }^{15} \mathrm{e}$ uma banda longitudinal ( $>600 \mathrm{~nm}$ ) como resultado da absorção induzida pelo eixo longo dos nanobastões, a qual varia de acordo com sua relação de aspecto (comprimento/largura). Com o aumento dessa razão, que pode ser determinado tanto pela modificação do tamanho da nanopartícula quanto pela ligação de moléculas em sua superfície, a cor do ouro em solução é alterada do azul para o vermelho e o comprimento de onda de ressonância de plasmon é deslocado. ${ }^{16-19}$

A existência da banda de absorção plasmônica longitudinal apresenta-se como o principal diferencial e grande vantagem dos nanobastões frente às nanoesferas, no que diz respeito à sua aplicação 
como sensores. A ligação de uma molécula ao nanobastão causa uma ligeira mudança no ambiente dielétrico próximo e consequentemente um desvio no pico de absorção da banda longitudinal, alteração que pode ser facilmente observada por meio da análise espectrofotométrica. ${ }^{14,19}$ Assim, as medidas de absorção óptica podem ser realizadas nas suspensões coloidais de nanobastões puros e acoplados a diversos ligantes e/ou agentes biológicos. O mesmo não é possível com nanoesferas de ouro, já que possuem apenas uma banda de absorção plasmônica que não sofre deslocamento após tais interações. Portanto, os nanobastões de ouro oferecem alta sensibilidade de absorção óptica após qualquer ligação física ou química na sua superfície, alterando de maneira mensurável o pico de absorção óptica relacionado ao modo longitudinal. ${ }^{12,14,17}$

Para que as nanopartículas de ouro apresentem aplicabilidade, o CTAB empregado na síntese deve ser removido ou substituído antes das etapas de funcionalização química com ligantes e/ou moléculas de interesse, tais como polietileno glicol (PEG), oligonucleotídeos, peptídeos, polímeros ou sílica inorgânica. ${ }^{14,19}$ Moléculas bi-funcionais são amplamente empregadas (Tabela 1), e geralmente possuem grupos de ácido carboxílico que atuam como ligantes químicos para bioconjugação através de acoplamento mediado por carbodiimida via amina primária. ${ }^{20}$

Tabela 1. Principais ligantes empregados para funcionalizar nanoesferas e nanobastões de ouro

\begin{tabular}{lc}
\hline Ligante & Referências \\
\hline PEG/ SH-PEG & 21 \\
Estreptavidina/Biotina & 22 \\
Aminoácidos & 23 \\
Precursores de sais de diazônio & 24 \\
Ácido 3-mercaptopropiônico (MPA) & 25 \\
Ácido 11-mercaptoundecanóico (MUDA) & 26 \\
Ácido lipóico & 27 \\
Polietilenoimina (PEI) & 28 \\
Cisteamina & 29 \\
\hline
\end{tabular}

Todos os eventos de funcionalização descritos acima podem ser facilmente monitorados através da ressonância plasmônica de superfície, já que a ligação de biomoléculas na superfície das nanopartículas altera o ambiente circundante e afeta a densidade de cargas dos elétrons ali presentes, o que acarreta em deslocamento da banda de absorção longitudinal. ${ }^{30}$ Nesse contexto, existem inúmeras aplicações baseadas nas alterações da ressonância plasmônica de superfície. Uma delas é o biosensoriamento, conhecido por nanoSPR, obtido pela ligação de biomoléculas (como anticorpos) aos nanobastões já acoplados a um ligante. A posterior interação com o alvo conclui a detecção, resultando em um deslocamento observável da banda longitudinal. ${ }^{19}$ Estes nanomateriais, portanto, podem ser empregados em ensaios de biosensoriamento químico, com fácil detecção do resultado final por meio da verificação do deslocamento da banda de absorção plasmônica longitudinal em espectrofotômetro. ${ }^{7}$

Trabalhos contendo propostas para introdução da nanociência em cursos de graduação envolvendo nanopartículas sintetizadas a partir de diferentes materiais (incluindo pontos de carbono, ouro e prata) já foram documentados. ${ }^{31-33}$ Alguns autores demonstraram a aplicabilidade de aulas práticas sobre síntese de nanopartículas de prata e ouro ${ }^{32,33}$ Nesses trabalhos, além dos métodos de síntese, foram abordadas as principais propriedades ópticas - dependentes do formato e tamanho - e catalíticas das nanopartículas de ouro. ${ }^{33}$
Recentemente foi reportada uma proposta de aula prática sobre nanopartículas de ouro depositadas em filmes de biopolímeros, na qual os alunos puderam verificar a atividade antimicrobiana da nanopartícula. ${ }^{34}$ No entanto, apesar da relativa facilidade com que as nanopartículas de ouro podem ser preparadas e modificadas quimicamente, não há ainda relatos da utilização destes nanomateriais em aulas práticas com foco em aplicações biotecnológicas. A experiência prática de sintetizar e manipular estes nanomateriais seria de grande relevância na formação acadêmica de estudantes de pós-graduação para a expansão e consolidação do conhecimento teórico e prático da nanotecnologia no Brasil.

Além de envolver conceitos de química (como teorias de ligação metálica, estrutura atômica, reações de oxidação-redução e espectroscopia), a prática experimental em nanotecnologia utilizando AuNPs como biosensores envolve também conceitos bioquímicos como interações antígeno-anticorpos e conformação de moléculas. Este caráter interdisciplinar agrega conhecimento aos alunos e valor aos cursos, já que a nanotecnologia é uma nova área que vem ganhando espaço no Brasil e no mundo.

Portanto, o presente trabalho retrata a experiência de uma aula prática de funcionalização de nanobastões de ouro, realizada durante o III Curso de Verão em Biotecnologia na Universidade Federal de Ouro Preto (UFOP). Essa é uma iniciativa de divulgação da nanobiotecnologia, de significativa importância para o avanço tecnológico e valorização da pesquisa. Além disso, este trabalho descreve as etapas de acoplamento do ligante polietilenoimina (PEI) à superfície de nanobastões de ouro e posterior ligação com o anticorpo anti-Interleucina 6 (IL-6). A IL-6 é uma proteína pró-inflamatória, glicosilada com peso molecular de $26 \mathrm{kDa}$, identificada pela primeira vez em 1980, produzida por macrófagos, células dendríticas, algumas células TCD4+ (dos tipos Th2 e Th17) e também por células não imunes (fibroblastos e células endoteliais). ${ }^{35,36}$ Este é, portanto, um modelo para o desenvolvimento de novos ensaios de detecção de biomoléculas que podem ser aplicados à pesquisa e ao ensino, na formação de pós graduandos em química e em biotecnologia.

\section{PARTE EXPERIMENTAL}

\section{Reagentes}

Para a síntese de nanopartículas de ouro foram utilizados os reagentes ácido tetracloroáurico 99\% (Sigma-Aldrich), brometo de cetiltrimetilamônio P.A. (CTAB, Sigma-Aldrich), ácido ascórbico e nitrato de prata, ambos com pureza mínima de 99\% (Aldrich). As nanopartículas foram funcionalizadas com o ligante polietilenoimina $50 \%$ w/v em água (Sigma-Aldrich). Foi utilizado o par anticorpo-antígeno do kit para ELISA de IL-6 humana (BD OptEIA ${ }^{\mathrm{TM}}$ ). Os anticorpos foram suspendidos em tampão fosfato salino (PBS), em cloridrato de N-etil-N'-(3-dimetil-aminopropil)carbodiimida P.A. (Sigma-Aldrich) e em N-hidroxisuccinimida 98\% (Sigma-Aldrich). Para bloqueio da superfície dos nanobastões, foi utilizada a albumina sérica bovina com pureza mínima de $98 \%$ (GE, Sigma). Todas as soluções aquosas foram preparadas com água deionizada e filtrada $(18 \mathrm{M} \Omega)$ e as vidrarias lavadas exaustivamente com ácido nítrico $65 \%$ (Vetec).

\section{Síntese de nanobastões de ouro}

As nanopartículas de ouro foram sintetizadas pelo método mediado por semente. ${ }^{13,37}$ Nesta síntese, as sementes de ouro foram preparadas por meio da mistura de $2,25 \mathrm{~mL}$ de $\mathrm{HAuCl}_{4}(5 \mathrm{mmol}$ $\left.\mathrm{L}^{-1}\right), 1,25 \mathrm{~mL}$ de CTAB $\left(0,2 \mathrm{~mol} \mathrm{~L}^{-1}\right)$ e $0,9 \mathrm{~mL}$ de solução resfriada de $\mathrm{NaBH}_{4}\left(1 \mathrm{mmol} \mathrm{L}^{-1}\right)$ sob agitação contínua, resultando em uma 
solução marrom-amarelada. A solução de crescimento foi obtida misturando-se $5 \mathrm{~mL}$ de solução $\mathrm{HAuCl}_{4}\left(1 \mathrm{mmol} \mathrm{L}^{-1}\right)$ com $5 \mathrm{~mL}$ de CTAB $\left(0,2 \mathrm{~mol} \mathrm{~L}^{-1}\right)$ e $\mathrm{AgNO}_{3}\left(4 \mathrm{mmol} \mathrm{L}^{-1}\right)$. Então, 0,07 mL de ácido ascórbico $\left(0,0788 \mathrm{~mol} \mathrm{~L}^{-1}\right)$ foram adicionados durante agitação vagarosa. Nessa etapa o ácido ascórbico age como agente redutor e altera a cor da solução de amarelo intenso para transparente. Por fim, $0,012 \mathrm{~mL}$ da solução semente foram adicionados à solução recém-preparada para iniciar o crescimento dos nanobastões. A cor da solução se alterou gradualmente entre os primeiros 15-20 min, até se estabilizar em tom arroxeado. Os nanobastões foram purificados retirando-se o excesso de CTAB por dupla centrifugação a $5.600 \mathrm{~g}$ por 15 min e redispersos em água ultrapura deionizada.

\section{Funcionalização de nanobastões de ouro}

Para permitir o acoplamento dos anticorpos, foi realizada a ligação de PEI à superfície dos nanobastões de ouro. Esse reagente é capaz de mediar a ligação covalente nanobastão-anticorpo devido aos vários grupos amina reativos. ${ }^{38}$ Para isso, $250 \mu \mathrm{L}$ de solução de nanobastões de ouro foram centrifugados a $4000 \mathrm{~g}$ por $10 \mathrm{~min}$, ressuspendidos em $250 \mu \mathrm{L}$ de PEI $(0,3 \%$ em água ultra pura $)$ e incubados a temperatura ambiente em banho ultrassônico por $30 \mathrm{~min}$, para desestabilização do CTAB presente na superfície das nanopartículas e acoplamento do PEI. Em seguida, a solução foi novamente centrifugada a $4000 \mathrm{~g}$ por $10 \mathrm{~min}$ e ressuspendida em $250 \mu \mathrm{L}$ de anticorpos anti-IL-6 a $0,4 \mu \mathrm{g} \mathrm{mL} \mathrm{L}^{-1}$ previamente preparados.

A solução dos anticorpos foi preparada adicionando-se $18,7 \mu \mathrm{L}$ de EDAC/HNS (0,4 mol L-1 / 0,1 mol L $\left.\mathrm{m}^{-1}\right), 0,4 \mu \mathrm{g} \mathrm{mL}^{-1}$ dos anticorpos anti-IL-6 e tampão fosfato salino (PBS 1X, pH 7.4, NaCl, KCl, $\mathrm{Na}_{2} \mathrm{HPO}_{4}, \mathrm{KH}_{2} \mathrm{PO}_{4}$ em água ultra pura) para um volume final de $250 \mu \mathrm{L}$ e, em seguida, incubada por $30 \mathrm{~min}$ a $4{ }^{\circ} \mathrm{C}$. Por fim, a reação de acoplamento dos nanobastões funcionalizados ao PEI com os anticorpos foi realizada por $60 \mathrm{~min}$ à temperatura ambiente em banho ultrassônico. Ao final deste tempo foram novamente centrifugadas a $4000 \mathrm{~g}$ por $10 \mathrm{~min}$ e ressuspendidas em $100 \mu \mathrm{L}$ de PBS contendo 62 $\mu \mathrm{g} \mathrm{mL} \mathrm{mL}^{-1}$ de BSA, para realizar o bloqueio de possíveis regiões livres remanescentes na superfície dos nanobastões de ouro. Transcorridos 30 min de incubação à temperatura ambiente, a solução foi centrifugada a $4000 \mathrm{~g}$ por 10 min e ressuspendida em $100 \mu \mathrm{L}$ de PBS contendo $100 \mathrm{pg} \mathrm{mL}^{-1}$ do antígeno específico IL-6.

\section{Instrumentação}

Os espectros de absorção plasmônica das soluções coloidais foram obtidos em um espectrofotômetro Shimadzu UV-1800, utilizando-se cubetas de quartzo de $1 \mathrm{~cm}$ de caminho óptico. As amostras foram também analisadas por leitura de potencial zeta, com o qual é possível aferir estabilidade e comprovar a ligação de moléculas nas nanopartículas, através da modificação da sua carga superficial quando dessa interação. Para as leituras, $10 \mu \mathrm{L}$ de amostra ressuspendida em PBS foram diluídos em 1,5 mL de água ultra pura e então, aplicados em cubetas específicas. As medidas foram feitas utilizando Zetasizer (NanoSeries - Malvern). Além disso, os dados resultantes da leitura em espectrômetro UV-Vis-NIR foram normalizados utilizando o software PeakFit V.4 e os gráficos foram gerados em GraphPad Prism $^{\circledR}$ e Origin v. 8.0.

\section{Segurança e riscos}

Todos os procedimentos experimentais são de simples execução e não expõem os alunos a riscos, desde que sejam realizados seguindo-se as normas básicas de biossegurança.

Deve ser dada atenção especial aos seguintes reagentes:
- PEI, ácido tetracloroáurico, borohidreto de sódio, NHS e EDAC: causam irritação na pele e olhos. Portanto, utilize luvas de proteção e vestuário de proteção (jaleco) durante seu manuseio. Caso entre em contato com os olhos, enxague continuamente com água corrente, remova lentes de contato e procure atendimento médico.

- Brometo de cetiltrimetilamônio (CTAB) é altamente tóxico para o sistema respiratório quando inalado. Portanto, utilize máscara durante sua pesagem. Em caso de ingestão acidental, enxague imediatamente a boca e procure atendimento médico.

- Nitrato de prata e do ácido nítrico são reagentes que causam irritação na pele e nos olhos, além de serem tóxicos quando inalados. Seu manuseio também requer a utilização de luvas de proteção, vestuário de proteção (jaleco) e máscara. Além disso, deve-se manter estes reagentes longe de materiais combustíveis e afastados do calor, faíscas, chamas e/ou superfícies quentes.

\section{RESULTADOS E DISCUSSÃO}

\section{Síntese de nanobastões de ouro}

O procedimento de síntese permitiu a obtenção de uma solução coloidal de cor arroxeada altamente estável de nanobastões de ouro. Tal como na síntese de nanoesferas, a formação dos nanobastões envolve a redução de $\mathrm{AuCl}^{4-}$ para $\mathrm{Au}^{0}$ pela ação do borohidreto de sódio, e, em seguida do ácido ascórbico, que atuam como agentes redutores. $^{32}$

O processo de síntese de nanobastões mediado por sementes consiste no alongamento assimétrico de nanoesferas de ouro (sementes) em apenas uma direção, devido à interação com o surfactante CTAB e íons de prata. Diferentes teorias tentam explicar tal fenômeno de crescimento assimétrico direcionado. ${ }^{39,40}$ Basicamente, os átomos de ouro encontram-se organizados em uma estrutura cúbica de face centrada e é suposto que a deposição de outros átomos de ouro (presentes na solução de crescimento) cause a quebra de assimetria dessa estrutura e o início do crescimento na direção em que ocorreu essa deposição inicial. ${ }^{12,14}$ A partir desse ponto, essa diferença estrutural acarreta em diferenças conformacionais e faces com diferentes energias de superfície, fato que, por sua vez, é responsável pela interação preferencial do surfactante (e dos íons de prata) com regiões específicas do bastão em crescimento. ${ }^{14,19} \mathrm{~A}$ face em que o crescimento foi iniciado apresenta um espaçamento entre os átomos que é mais propício à interação do $\mathrm{CTA}^{+}$do que com as demais. Por outro lado, as demais faces apresentam maior energia de superfície, a qual é estabilizada pela interação com o CTAB. Na solução de crescimento, os átomos de ouro encontram-se na forma de $\mathrm{AuCl}_{2}{ }^{-}$ligado à micelas de $\mathrm{CTAB}$. A interação entre os campos elétricos do $\mathrm{AuCl}_{2}^{-}$(carregado negativamente) e da semente em crescimento (carregada positivamente) faz com que as colisões entre as partículas sejam mais frequentes na extremidade em que o crescimento foi iniciado, direcionando o alongamento em forma de bastão. ${ }^{14}$ Além do papel exercido pelo CTAB, os íons de prata também são importantes no controle da síntese por exercerem duas atividades fundamentais. ${ }^{12,19} \mathrm{~A}$ primeira consiste em diminuir a densidade de carga dos íons brometo e reduzir a repulsão entre as moléculas de CTAB ligadas às faces não-crescentes da semente, aprimorando a forma dos bastões. A segunda função exercida pelos íons de prata é a limitação do tamanho dos bastões. Isso ocorre devido à redução dos íons $\mathrm{Ag}^{+}$a $\mathrm{Ag}^{0}$ que ocorre na presença de outros metais (como o ouro) e posterior deposição lenta nas faces em que houve alongamento, limitando o comprimento dos bastões a aproximadamente $100 \mathrm{~nm} . .^{14,41}$

Ao final das etapas da síntese os alunos observaram que a solução resultante apresentou uma coloração arroxeada, e desta solução 
foi separada uma alíquota para a análise espectroscópica na região UV-Vis-NIR (Figura 1A). Além disso, quando disponível, os alunos podem realizar imagem por microscopia eletrônica de transmissão (TEM) para visualização do material sintetizado (Figura 1B). Previamente à realização do experimento, os alunos não consideravam a hipótese do ouro assumir diferentes cores. Os alunos responderam em questionário que o ouro certamente possui cor amarela, podendo assumir diferentes tonalidades apenas quando associado a outros metais. Nenhum aluno fazia ideia da maneira pela qual poderiam ser sintetizadas nanopartículas de ouro em laboratório e ficaram satisfeitos com o resultado e aprendizado, principalmente com a variedade de cores que as nanopartículas metálicas podem assumir. Dessa forma, esse experimento possibilitou a discussão dos conceitos de ressonância plasmônica de superfície e confinamento quântico, responsáveis, respectivamente, pela origem e variedade de cores das nanopartículas em função do tamanho. ${ }^{32}$

\section{Montagem do biosensor}

Os anticorpos anti-IL-6 foram acoplados aos nanobastões de ouro por intermédio do reagente polietilenoimina (PEI), que se liga eletrostaticamente à superfície do ouro. Tal etapa é de suma importância para que ocorra a ligação covalente de um dos grupos amina $\left(-\mathrm{NH}_{2}\right)$ expostos no PEI com o grupo carboxila (-COOH) presente na porção C-terminal do anticorpo. As imunoglobulinas da classe $\mathrm{G}$ (IgG) são constituídas de duas cadeias pesadas, unidas entre si por pontes dissulfeto, e duas cadeias leves, unidas às cadeias pesadas também por pontes dissulfeto (Figura 2). Quando digeridas pela enzima papaína, as IgGs são separadas em uma região constante (Fc), que determina as funções efetoras do anticorpo, e duas regiões variáveis (Fab), responsáveis pela especificidade de ligação para cada antígeno. ${ }^{42}$ Durante a ligação ao reagente PEI, essas regiões devem permanecer livres para permitir o posterior acesso do antígeno. Por
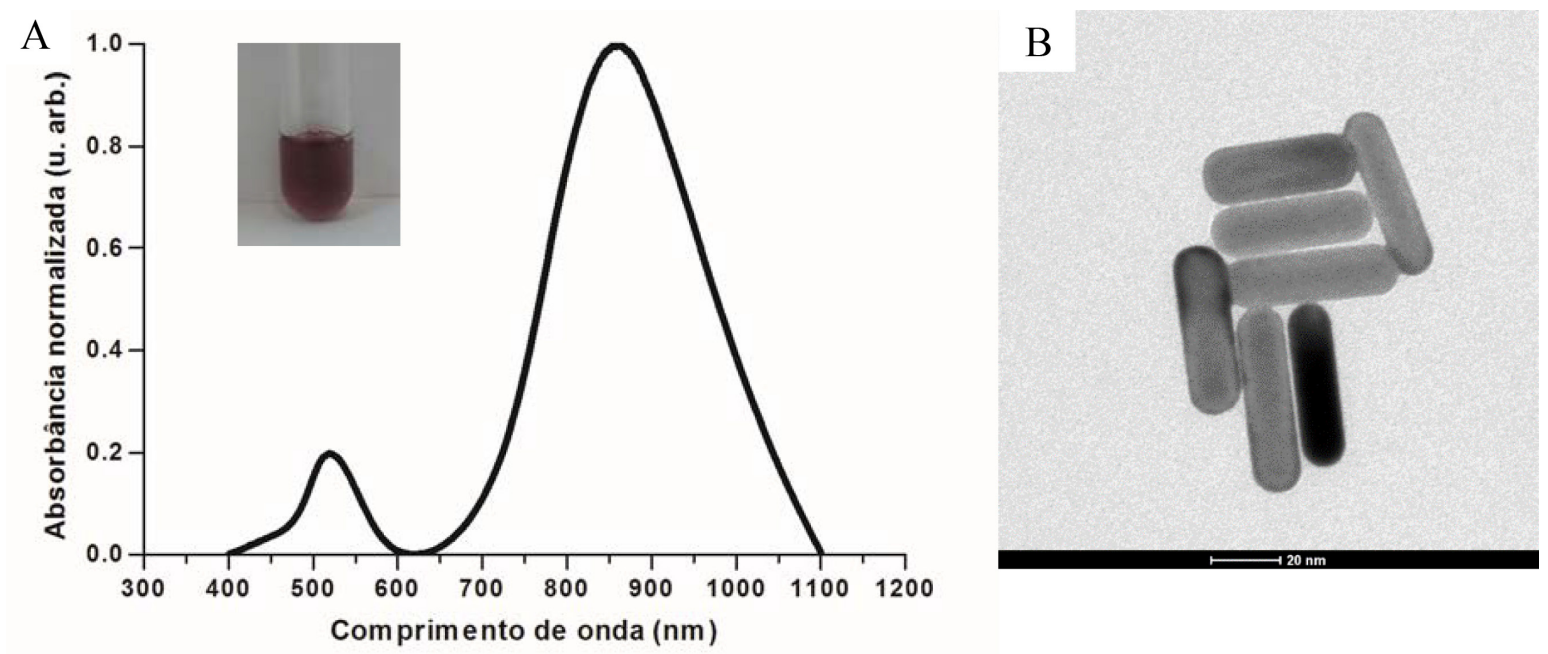

Figura 1. A: Espectro de absorção na região do ultravioleta-visível-infravermelho próximo (UV-Vis-NIR), com picos de absorção plasmônica transversal $(520 \mathrm{~nm})$ e longitudinal $(863 \mathrm{~nm}$ ). Inserção: aspecto visual da solução de nanobastões de ouro. B: Imagem de microscopia eletrônica de transmissão (TEM) representativa de um resultado de síntese através do protocolo aqui descrito

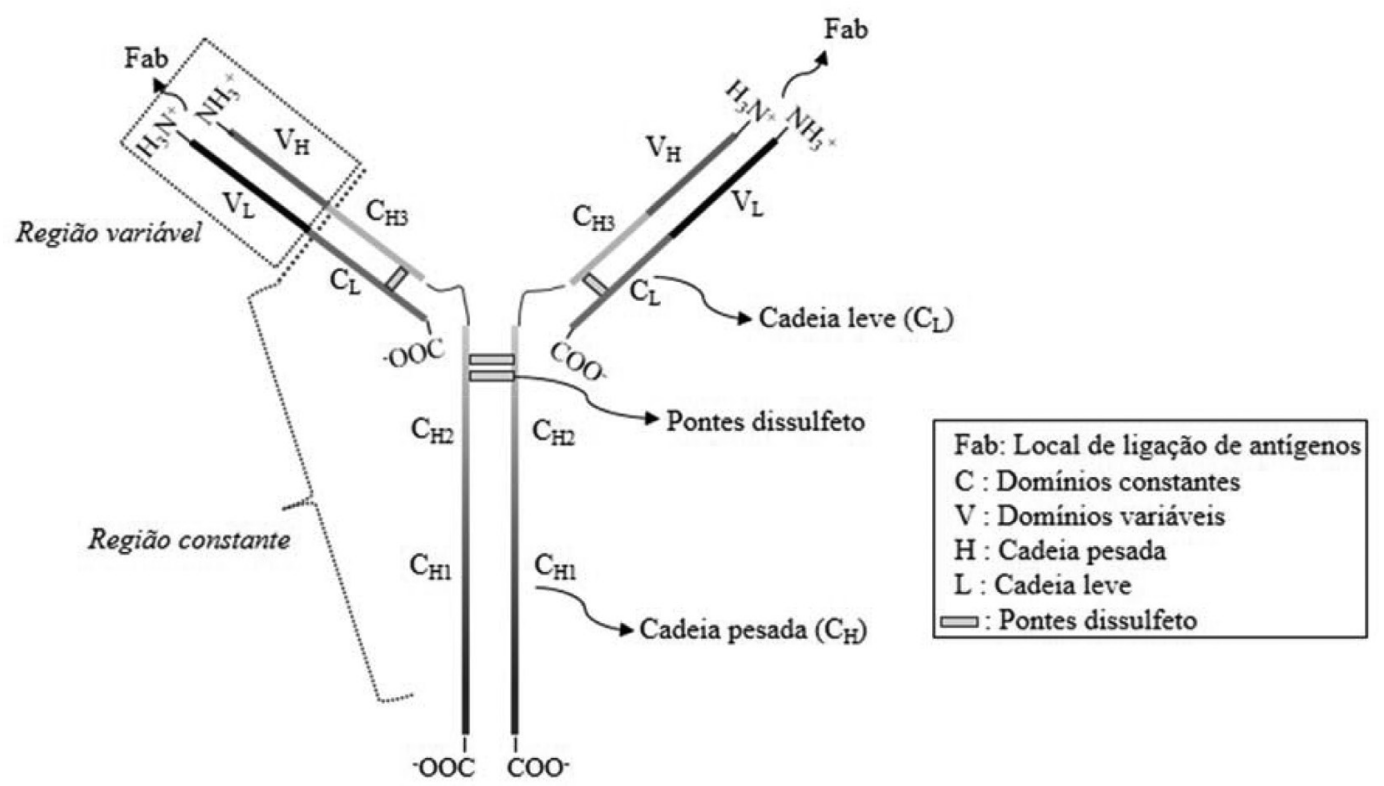

Figura 2. Estrutura tridimensional básica de um anticorpo. Cada cadeia pesada $\left.{ }_{H}\right)$ e leve $\left(_{L}\right)$ possui uma região variável $(V)$ e uma região constante $(C)$. As duas cadeias pesadas são unidas entre si e entre uma cadeia leve por pontes dissulfeto. A porção C-terminal contendo grupos carboxila está localizada na região constante das cadeias pesadas e a porção $\mathrm{N}$-terminal contendo grupos amina situa-se na região variável que engloba ambas as cadeias leves e pesadas 

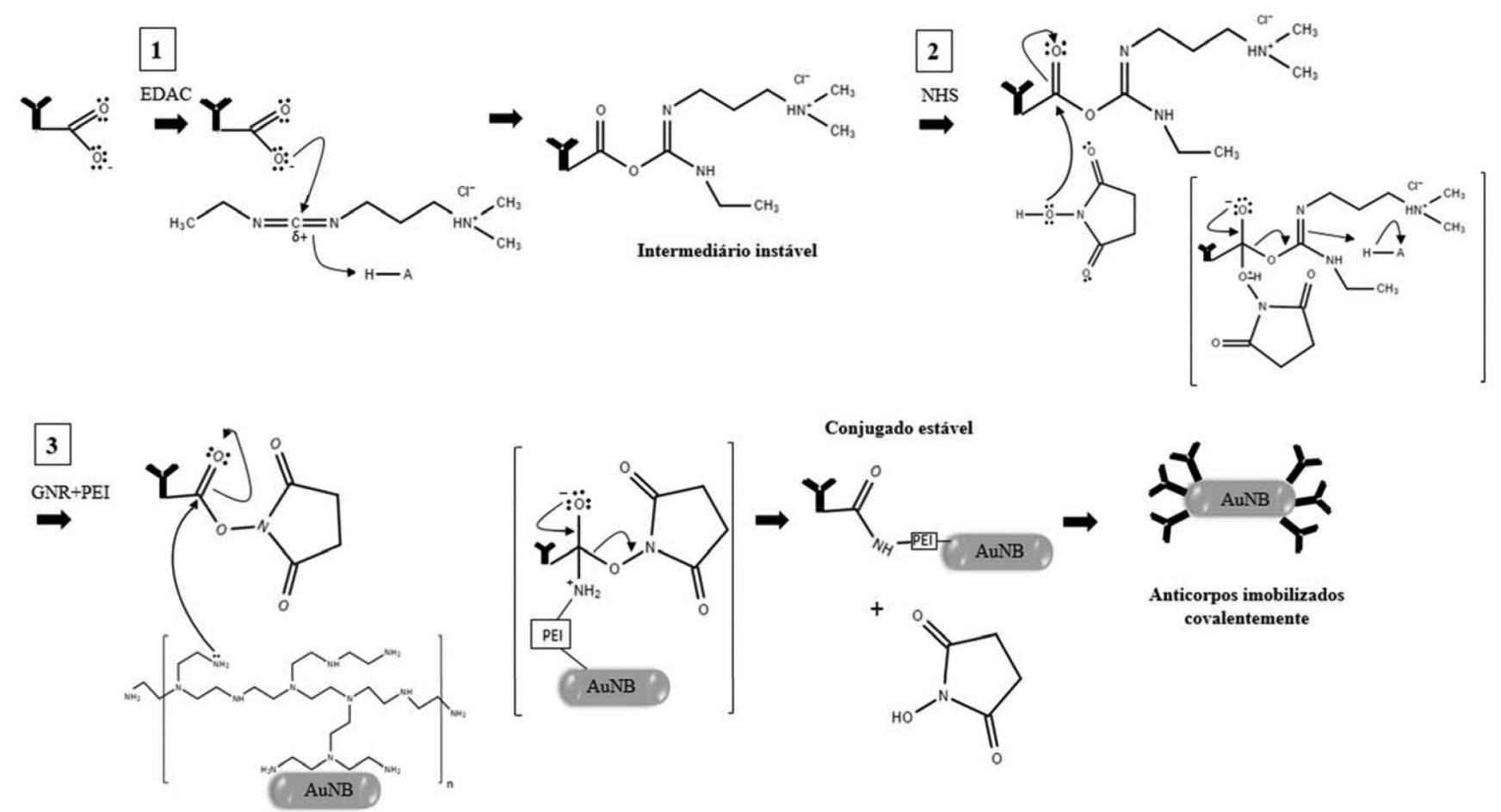

Figura 3. Esquema de reação de "crosslinking" entre PEI e o anticorpo anti-IL-6 empregando EDAC e NHS. 1: Reação de adição eletrofílica do EDAC ao anticorpo. 2: Substituição nucleofílica do EDAC por NHS, formando um éster de NHS. 3: Reação entre o anticorpo contendo o éster de NHS e a amina primária do PEI para formação da ligação amida. AuNB: Nanobastões de ouro

esse motivo, a ligação é estabelecida com os grupos carboxila das cadeias pesadas das IgGs, localizadas na extremidade da porção Fc.

A ligação amida do grupo carboxila presente na porção C-terminal do anticorpo com os grupos amina do PEI é obtida por meio de uma reação de acoplamento ("crosslinking"), realizada com o emprego de um agente acoplante (N-etil-N'-(3-dimetil-aminopropil) carbodiimida - EDAC) e um agente estabilizante (N-hidroxisuccinimida - NHS). Ambos reagentes servem como ativadores do grupo carboxila. A despeito do uso isolado do EDAC já resultar em um composto com a capacidade de formar uma ligação amida, a reação entre esse composto e o NHS forma um éster com menor probabilidade de sofrer hidrólise, aumentando a probabilidade da reação entre o C-terminal ativado e a amina primária do PEI. As reações ocorridas em cada etapa são mostradas na Figura 3. Além disso, a Figura 4 resume as principais etapas de síntese e funcionalização dos nanobastões de ouro, necessárias para a construção do biosensor no desenvolvimento da aula prática.

A ligação de moléculas na superfície dos nanobastões altera o ambiente circundante e afeta a densidade de cargas dos elétrons ali presentes, acarretando em um deslocamento da banda de absorção longitudinal. ${ }^{5}$ Dessa forma, após adição do reagente polietilenoimina (PEI) à solução contendo nanobastões de ouro, pôde ser observado um deslocamento de $62 \mathrm{~nm}$ para a esquerda (blue-shift), comprovando a ligação. O blue-shift do pico de absorção plasmônica longitudinal indica que houve uma diminuição da constante dielétrica no meio circundante. Em seguida, verificou-se a efetiva ligação do anticorpo anti-IL-6 ao PEI, sendo observado um deslocamento de $29 \mathrm{~nm}$ para a direita (red-shift), devido ao aumento do índice de refração do ambiente circundante. Ambos os deslocamentos podem ser facilmente visualizados na Figura 5, comprovando a ligação do linker PEI (linha tracejada) e o posterior acoplamento do anticorpo anti-IL6 (linha pontilhada).

Posteriormente, considerando que a proteína modelo albumina sérica bovina (BSA) possui capacidade de se adsorver facilmente à
Formação de sementes através da redução de $\mathrm{AuCl}_{4}{ }^{-}$para $\mathrm{Au}^{0}$

Controle do crescimento em forma de bastões pela adição de CTAB, o qual se liga preferencialmente nas faces laterais das sementes

$$
\checkmark
$$

Armazenamento do frasco contendo os nanobastões de ouro a $4{ }^{\circ} \mathrm{C}$ até o uso Y

Solubilização de eventuais grânulos através da imersão do frasco em béquer contendo água aquecida $\left(\cong 40^{\circ} \mathrm{C}\right)$ previamente à realização dos experimentos

Desestabilização e remoção de parte do $\mathrm{CTAB}$ através de centrifugação $\checkmark$

Adição do ligante PEI à solução: substituição do CTAB pelo ligante através de sonicação

$\checkmark$

Remoção de PEI não ligado através de centrifugação

Adição de biomoléculas à solução

Remoção de biomoléculas não ligadas através de centrifugação

Incubação do biosensor com água ou amostra contendo a molécula alvo

Leitura espectrofotométrica na região UV-Vis-NIR: aliquotas de $100 \mu \mathrm{L}$ submetidas à varredura na faixa de 400 a $1000 \mathrm{~nm}$

Figura 4. Principais etapas de síntese e funcionalização de nanobastões de ouro para desenvolvimento da aula prática utilizando biosensor

superfície de nanopartículas de ouro ${ }^{43}$, os nanobiosensores foram incubados com BSA $\left(62 \mu \mathrm{g} \mathrm{mL}^{-1}\right)$ para permitir o bloqueio de possíveis sítios dos nanobastões que não tenham sido ocupados. Nesta reação, houve um deslocamento de apenas $4 \mathrm{~nm}$ para a esquerda, em relação à banda de absorção plasmônica longitudinal de nanobastões 


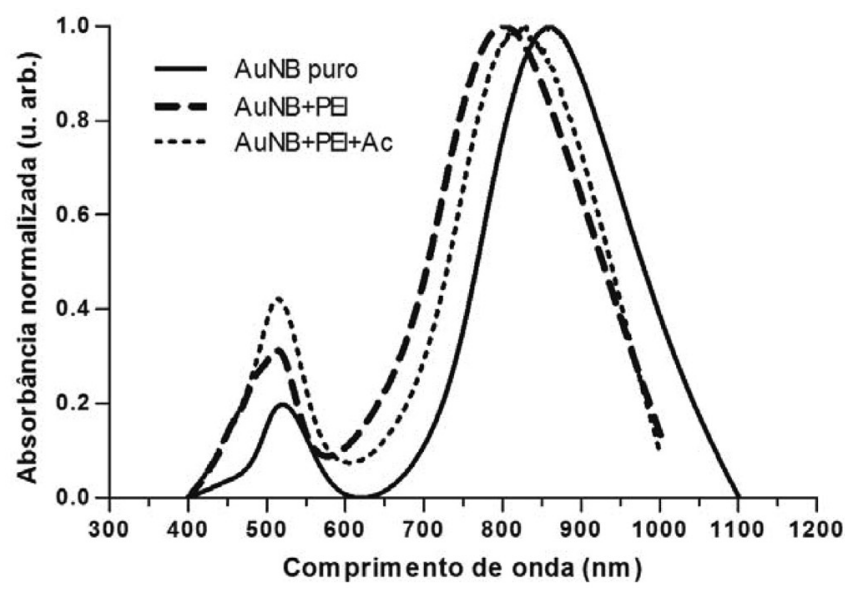

Figura 5. Espectros de absorção óptica da funcionalização de nanobastões de ouro (AuNBs). Espectro resultante de AuNBs puros com pico máximo de absorção plasmônica longitudinal de $863 \mathrm{~nm}$ em linha contínua; ligação de PEI (0,3\%) aos AuNBs com pico máximo de absorção plasmônica longitu-

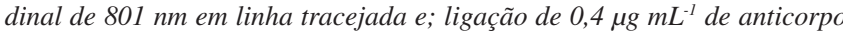
anti-IL-6 com pico máximo de absorção plasmônica longitudinal de $830 \mathrm{~nm}$ em linha pontilhada

já ligados ao PEI e aos anticorpos (Figura 6). Esse deslocamento não é significativo no que concerne ao efeito de biosensoriamento, mas sugere que o BSA tenha sido adsorvido aos locais nos quais o reagente PEI não havia se ligado. Além disso, após novas adições de BSA, não ocorre deslocamento, o que corrobora a hipótese do completo bloqueio. A ocupação de possíveis sítios remanescentes impede posteriores ligações inespecíficas na superfície dos nanobastões, que poderiam ocasionar resultados falsos na etapa do biosensoriamento. Dessa forma, a finalização de tais etapas resultam na completa funcionalização dos nanobastões de ouro, que podem ser, a partir de então, considerados nanobiosensores.

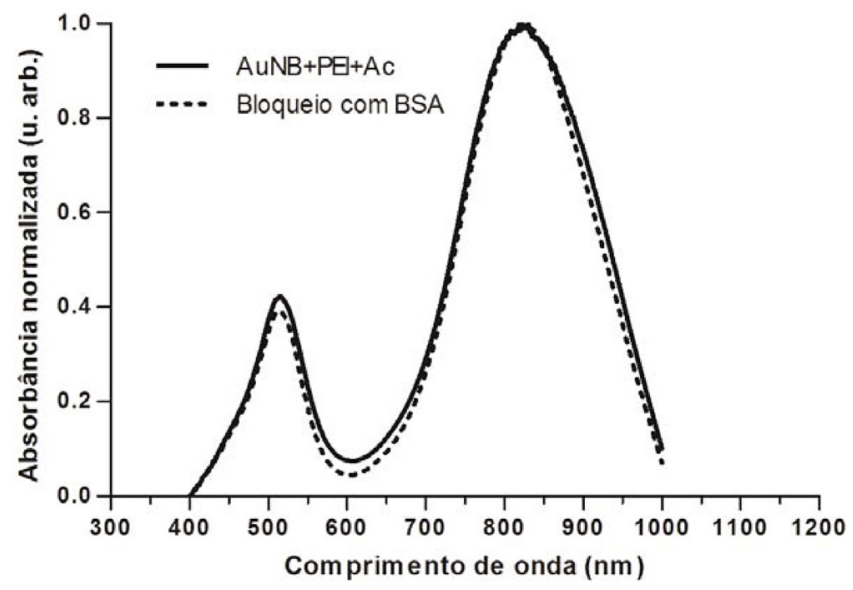

Figura 6. Espectros de absorção óptica do bloqueio de nanobastões de ouro com a proteína albumina bovina sérica (BSA). Espectro resultante de nanobastões puros ligados ao PEI $(0,3 \%)$ e à $0,4 \mu \mathrm{g} \mathrm{mL}^{-1}$ de anticorpo anti-IL-6 com pico máximo de absorção plasmônica longitudinal de $830 \mathrm{~nm}$ em linha contínua e; ligação de $62 \mu \mathrm{g} \mathrm{mL} L^{-1}$ de BSA aos nanobastões com pico máximo de absorção plasmônica longitudinal de $826 \mathrm{~nm}$ em linha tracejada

\section{Detecção nanosonda-alvo}

Após a etapa de preparo da nanosonda, a superfície dos nanobastões encontra-se recoberta por anticorpos ligados e os sítios livres bloqueados com BSA. Dessa forma, o nanosensor encontra-se apropriado para os eventos de interação com o antígeno alvo. Portanto, a solução de nanobastões de ouro foi incubada com o antígeno específico IL-6 (100 $\left.\mathrm{pg} \mathrm{mL}^{-1}\right)$ e sua interação efetiva com o anticorpo resultou em deslocamento significativo de $25 \mathrm{~nm}$ para a direita, como pode ser visualizado na Figura 7.

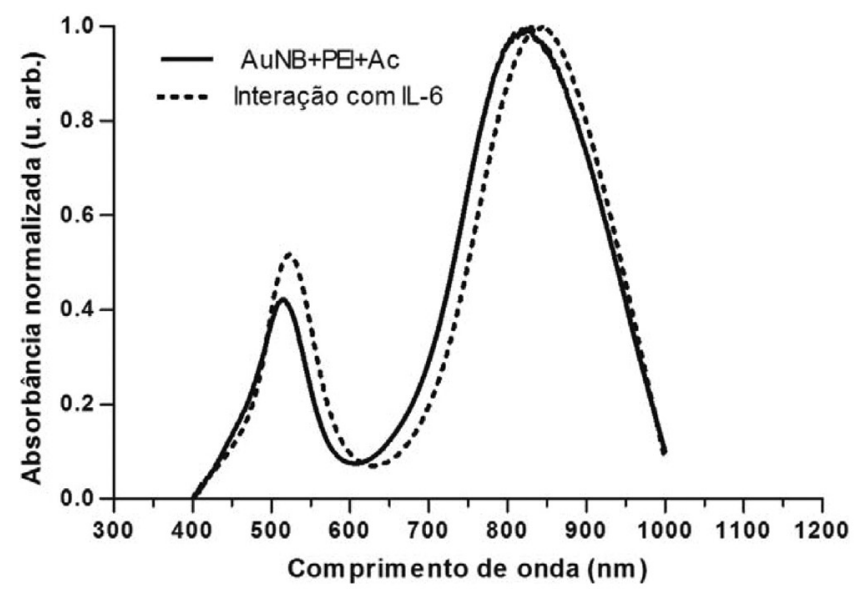

Figura 7. Espectros de absorção óptica do biosensoriamento de AuNBs. Espectro resultante do nanobiosensor com pico máximo de absorção plasmônica longitudinal de $826 \mathrm{~nm}$ em linha contínua e; ligação de $100 \mathrm{pg} \mathrm{mL}^{-1}$ de IL-6 aos AuNBs com pico máximo de absorção plasmônica longitudinal de $851 \mathrm{~nm}$ em linha tracejada

A visualização desse deslocamento na banda de absorção plasmônica longitudinal finaliza o experimento, comprovando de maneira simples uma interação sonda-alvo. Além de verificar a ligação de moléculas/biomoléculas na superfície dos nanobastões por meio do deslocamento do comprimento de onda da banda de absorção plasmônica longitudinal, existem outras maneiras que confirmam o biosensoriamento. Dessa forma, todas as reações de troca de ligantes ou detecção de analitos podem ser avaliadas também pelo pico Raman das biomoléculas (especialmente das ligações Au-S em cerca de 260 $\mathrm{cm}^{-1}$ ), pela estabilidade dos bioconjugados em solução tamponada ou ainda através de espectroscopia no infravermelho por transformada de Fourier (FTIR). As ligações químicas possuem modos vibracionais com assinaturas em comprimentos de onda específicos, e, dessa forma, as ligações envolvidas nas interações PEI-nanobastão e biosensor-antígeno podem ser comprovadas por meio da técnica de FTIR. ${ }^{44,45}$ Além disso, as interações podem também ser analisadas através de alterações do potencial zeta, o qual pode ser medido e avaliado em suspensões coloidais. ${ }^{14}$

O potencial zeta, ou potencial eletrocinético, é uma propriedade física exibida por qualquer partícula em suspensão, muito empregado na otimização de formulações de suspensões e emulsões, auxiliando também na predição da estabilidade a longo prazo. ${ }^{46}$ Dessa forma, o potencial zeta constitui uma medida tanto da estabilidade quanto da ligação de moléculas nas partículas, uma vez que os valores mais negativos ou positivos estão associados com soluções mais estáveis, já que a repulsão entre as nanopartículas reduz a agregação das

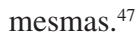

Após confirmação da interação sonda-alvo, visualizada através do deslocamento do pico de absorção plasmônica longitudinal por meio da espectroscopia na região UV-Vis-NIR, foi realizada leitura do potencial zeta. Devido à simplicidade de execução, o potencial zeta pode ser empregado, quando disponível, de maneira complementar para aprimorar a análise pelos estudantes e introduzir os conceitos básicos dessa técnica. Ressaltamos que a realização de uma aula prática somente com a visualização espectroscópica do deslocamento da banda de absorção plasmônica longitudinal é suficiente para que os alunos comtemplem a interação nanosonda-alvo. 
Os nanobastões de ouro são sintetizados com CTAB, uma molécula carregada positivamente que confere estabilidade. Após a ligação de outras moléculas na superfície das nanopartículas, parte deste CTAB é retirada e, então, a medida do potencial zeta deve ser reduzida. Isso acontece uma vez que a solução perde parte da estabilidade oferecida pelo CTAB. No caso de moléculas carregadas positivamente o valor do potencial zeta continua positivo, e em caso contrário, o valor se torna negativo. ${ }^{48}$

Portanto, quando o PEI é misturado aos nanobastões de ouro carregados positivamente, a carga líquida é diminuída. Da mesma forma, a cada nova ligação efetiva, a carga tende a diminuir, se negativando de maneira dependente da carga dos ligantes. Em todos os casos o valor do potencial zeta deve ser diferente de zero, garantindo a estabilidade. A Figura 8 mostra as leituras obtidas, em que houve uma diminuição dos valores em $\mathrm{mV}$ para os controles (AuNB+PEI+Ac; AuNB+Bloqueio com BSA), em relação à solução pura de nanobastões de ouro (AuNB). Na leitura após adição de BSA $\left(124 \mu \mathrm{g} \mathrm{mL}^{-1}\right)$, um alvo inespecífico, os valores de potencial zeta foram equiparados àqueles encontrados para o nanosensor, enquanto na presença de IL-6 o valor em mV se tornou negativo, com diferença estatística significativa em relação à nanosonda, comprovando sua detecção.

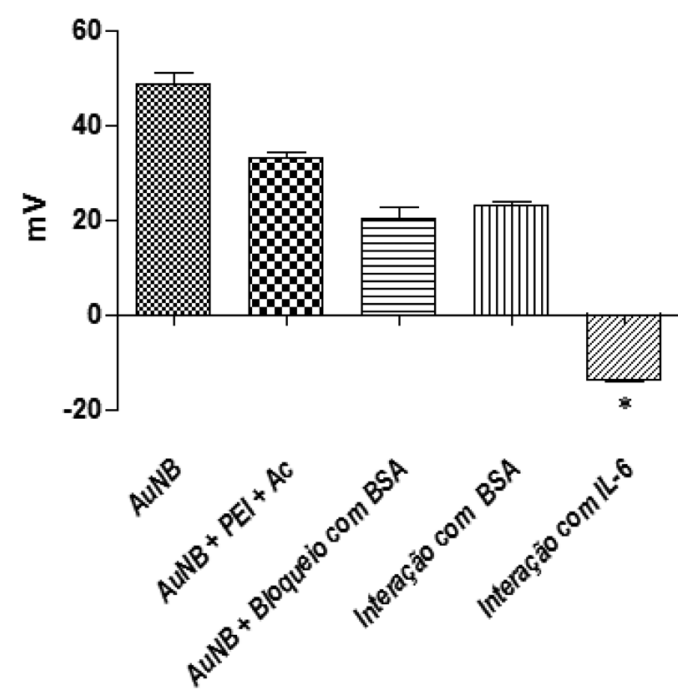

Figura 8. Potencial Zeta $(\mathrm{mV})$ para as diferentes condições de preparo e detecção do nanobiosensor. *intensidade em $m V$ significativamente diferente em relação ao nanobastão funcionalizado ao anticorpo anti-IL-6 $(p \leq 0,05)$. $n=10$

A análise do potencial zeta complementa a avaliação da interação nanosonda-alvo e fornece uma indicação da estabilidade do sistema coloidal, sendo útil para a introdução desse conceito aos estudantes. Se todas as nanopartículas em suspensão possuem valores altos de potencial zeta negativo ou positivo, elas tenderão a se repelir umas às outras e não haverá tendência para que as nanopartículas se unam. No entanto, se as nanopartículas apresentam baixos valores de potencial zeta, não haverá força para evitar que elas se agreguem. ${ }^{46}$ No caso do experimento de biosensoriamento, os alunos puderam verificar, através das leituras de potencial zeta, que houve ligação efetiva em cada etapa, sempre mantendo a estabilidade do sistema.

Ao término dos experimentos, os alunos responderam um questionário a respeito da experiência adquirida e suas perspectivas futuras para o emprego da nanotecnologia (Quadro 1). No âmbito da vida profissional, todos os alunos vislumbraram aplicabilidade das nanopartículas de ouro em suas pesquisas de iniciação científica, e se sentiram entusiasmados quanto à isso. Alguns exemplos incluíram novas formas de diagnóstico, detecção de substâncias no meio ambiente e direcionamento de drogas. De maneira geral, os alunos participantes do curso demonstraram vago conhecimento em relação ao tema e afirmaram que o curso lhes proporcionou um aprofundamento conceitual na área da nanotecnologia, com o qual foi possível uma melhor compreensão de todas as etapas da abordagem prática na síntese dos nanobastões de ouro.

Quadro 1. Perguntas contidas no questionário disponibilizado após o experimento em nanotecnologia realizado com os alunos do III Curso de Verão em Biotecnologia da UFOP

\begin{tabular}{|ll|}
\hline Perguntas realizadas \\
\hline 1. Já conhecia o assunto abordado? \\
2. O que o curso realizado lhe proporcionou? \\
3. Durante o curso você foi capaz de reformular conceitos à respeito \\
do assunto? \\
4. O assunto possui aplicação prática na sua vida profissional? \\
5. O material didático utilizado foi suficiente? \\
6. Você acha interessante a introdução de uma prática laboratorial com \\
nanopartículas de ouro em cursos de pós-graduação em química/ \\
biotecnologia?
\end{tabular}

As respostas do questionário aplicado foram satisfatórias e mostraram que a aula prática realizada desempenhou um impacto positivo na vida acadêmica dos participantes, uma vez que adquiriram o conhecimento multidisciplinar das diversas aplicações da nanotecnologia. Todos os participantes avaliaram o material didático como adequado e suficiente (apostila contendo o roteiro prático e os conceitos descritos nesse trabalho). Além disso, todos os alunos consideram que a introdução de aulas práticas envolvendo nanopartículas de ouro é de relevante importância para a formação profissional nos cursos de pós-graduação nas áreas de química e/ou biotecnologia.

$\mathrm{Na}$ esfera educacional, é fundamental que a interação dos alunos com o ambiente da pesquisa seja cada vez mais incentivada, com a aplicação prática do conhecimento teórico desenvolvido em sala. Isso demostra a conveniência dessa aula prática para a revisão de conceitos de química e bioquímica, sendo viável sua utilização em cursos de pós-graduação, nos quais os estudantes já são capazes de aplicar esses conceitos interdisciplinares para seu desenvolvimento técnico-científico. Consideramos que, uma vez seguidas as diretrizes contidas nesse trabalho, a utilização de nanobastões de ouro para aplicação em aulas práticas de nanotecnologia em maior escala será bem sucedida. Em vista disso, a síntese dos nanobastões de ouro, a posterior confecção do nanobiosensor, a comprovação da detecção do alvo específico por espectroscopia UV-Vis-NIR e a análise complementar do potencial zeta foram etapas realizadas satisfatoriamente e se mostraram capazes de estimular ainda mais o interesse dos alunos pela nanotecnologia.

\section{CONCLUSÃO}

A proposta de uma aula prática envolvendo nanobastões de ouro foi desenvolvida com êxito e os alunos demostraram interesse em se aprofundar nos diversos conceitos da nanotecnologia. A funcionalização dos nanobastões de ouro com polietilenoimina e anticorpos, e posterior detecção de IL-6, foi realizada por um grupo de quatro graduandos sob supervisão de um aluno da pós-graduação. A partir dos ensaios realizados e dos resultados alcançados, sugerimos que o biosensor proposto pode ser utilizado para o estabelecimento de uma ou duas aulas práticas, uma vez que é simples e multidisciplinar, abrangendo conhecimentos de física, biologia e principalmente química. Devido à dificuldade média no entendimento da prática, ela 
é proposta para alunos de pós-graduação, sendo factível e gerando muitos retornos para a formação do estudante. Essa abordagem contribui ainda para a divulgação da nanotecnologia e, consequentemente, para o avanço tecnológico e valorização da pesquisa nesta área. Por fim, poderá expandir horizontes aos estudantes, transmitindo novas perspectivas de pesquisas e campos de trabalho.

\section{AGRADECIMENTOS}

Aos alunos participantes do III Curso de Verão em Biotecnologia da UFOP, que participaram da aula prática em nanotecnologia e auxiliaram na obtenção dos resultados. Ao Laboratório de Nanomateriais do Departamento de Física da Universidade Federal de Minas Gerais e ao Laboratório Multiusuários da Escola de Farmácia da Universidade Federal de Ouro Preto. Às instituições de fomento FAPEMIG (projeto FAPEMIG CDS - APQ 01510-14), CAPES e CNPq.

\section{REFERÊNCIAS}

1. Hullmann, A.; Scientometrics 2007, 70, 739.

2. Hussein, A. K.; Renewable Sustainable Energy Rev. 2015, 42, 460.

3. Fangueiro, J. F.; Silva, A. M.; Garcia, M. L.; Souto, E. B.; Eur. J. Pharm. Biopharm. 2015, 95, 307.

4. Perret, G.; Ginet, P.; Tarhan, M. C.; Baccouche, A.; Lacornerie, T.; Kumemura, M.; Jalabert, L.; Solid-State Electron. 2016, 115, 66.

5. Daniel, M.-C.; Astruc, D.; Chem. Rev. 2004, 104, 293.

6. Pissuwan, D.; Valenzuela, S.; Cortie, M. B.; Biotechnol. Genet. Eng. Rev. 2008, 25, 93.

7. Wilson, R.; Chem. Soc. Rev. 2008, 37, 2028.

8. Jensen, T. R.; Malinsky, M. D.; Haynes, C. L.; VanDuyne, R. P.; J. Phys. Chem. B 2000, 104, 10549.

9. Santos, J. F. L.; Santos, M. J. L.; Thesing, A.; Tavares, F.; Griep, J.; Rodrigues, M. R. F.; Quim. Nova 2016, 39, 1098.

10. Faraday, M.; Phil. Trans. R. Soc. London 1857, 147, 145.

11. Turkevich, J.; Stevenson, P. C.; Hillier, J.; Anal. Chem. 1951, 55, 475.

12. Vigderman, L.; Khanal, B. P.; Zubarev, E. R.; Adv. Mater. 2012, 24, 4811.

13. Nikoobakht, B.; El-sayed, M. A.; Chem. Mater. 2003, 15, 1957.

14. Huang, X.; Neretina, S.; El-Sayed, M. A.; Adv. Mater. 2009, 21, 4880.

15. Huang, X.; El-Sayed, M. A.; J. Adv. Res. 2010, 1, 13.

16. El-Sayed, S. L. and M. A.; J. Phys. Chem. B 1999, 103, 8410.

17. Jain, P. K.; Huang, X.; El-Sayed, I. H.; El-Sayed, M. A.; Acc. Chem. Res. 2008, 41, 1578.

18. Chang, S.-S.; Lee, C.-L.; Wang, C. R. C.; J. Phys. Chem. B 1997, 101, 6661.

19. Yang, D.-P.; Cui, D.-X.; Chem. Asian J. 2008, 3, 2010.

20. Hwang, S.-Y.; Tao, A. R.; Pure Appl. Chem. 2011, 83, 233.

21. Wang, W.; Wei, Q.-Q.; Wang, J.; Wang, B.-C.; Zhang, S.-H.; Yuan, Z.; J. Colloid Interface Sci. 2013, 404, 223.

22. Hu, C.; Dou, W.; Zhao, G.; Electrochim. Acta 2014, 117, 239.

23. Wangoo, N.; Bhasin, K. K.; Mehta, S. K.; Suri, C. R.; J. Colloid Interface Sci. 2008, 323, 247.
24. Adabo, A. H.; Zeggari, R.; Said, N. M.; Bazzi, R.; Elie-caille, C.; Marquette, C.; Martini, M.; Tillement, O.; Perriat, P.; Chaix, C.; Boireau, W.; Roux, S.; J. Colloid Interface Sci. 2016, 467, 271.

25. Nietzold, C.; Lisdat, F.; Analyst 2012, 137, 2821.

26. Chen, S.; Zhao, Q.; Zhang, L.; Wang, L.; Zeng, Y.; Huang, H.; Sens. Actuators, B 2015, 221, 1391.

27. Mattoussi, H.; Mauro, J. M.; Goldman, E. R.; Anderson, G. P.; Sundar, V. C.; Mikulec, F. V; Bawendi, M. G.; J. Am. Chem. Soc. 2000, 122, 12142.

28. Pyshnaya, I. a.; Razum, K. V.; Poletaeva, J. E.; Pyshnyi, D. V.; Zenkova, M. a.; Ryabchikova, E. I.; Biomed Res. Int. 2014, 2014, 1.

29. Ma, Y.; Jiang, L.; Mei, Y.; Song, R.; Tian, D.; Huang, H.; Analyst 2013, 138,5338

30. Tiwari, P.; Vig, K.; Dennis, V.; Singh, S.; Nanomaterials 2011, 1, 31.

31. Vaz, R.; Vieira, K. O.; Machado, C. E.; Ferrari, J. L.; Schiavon, M. A.; Quim. Nova 2015, 38, 1366.

32. Junior, M. A. M.; Santos, L. S. S.; Gonçalves, M. do C.; Nogueira, A. F.; Quim. Nova 2012, 35, 1872.

33. Silva, A. G. M.; Rodrigues, T. S.; Macedo, A.; Silva, R. T. P.; Camargo, P. H. C.; Quim. Nova 2014, 37, 1716.

34. Quadros, C. C.; Brunelli, M. F.; Faria, V. W.; Scheeren, C. W.; Quim. Nova 2016, 39, 1015.

35. Hirano, T.; Int. Rev. Immunol. 1998, 16, 249.

36. Rincon, M.; Trends Immunol. 2012, 33, 571.

37. Xu, X.; Zhao, Y.; Xue, X.; Huo, S.; Chen, F.; Zou, G.; Liang, X.-J.; J. Mater. Chem. A 2014, 2, 3528.

38. Zapp, E.; Westphal, E.; Gallardo, H.; de Souza, B.; Cruz Vieira, I.; Biosens. Bioelectron. 2014, 59, 127.

39. Pérez-Juste, J.; Liz-Marzán, L. M.; Carnie, S.; Chan, D. Y. C.; Mulvaney, P.; Adv. Funct. Mater. 2004, 14, 571.

40. Murphy, C. J.; Sau, T. K.; Gole, A. M.; Orendorff, C. J.; Gao, J.; Gou, L.; Hunyadi, S.E.; Li, T.; J. Phys. Chem. B 2005, 109, 13857.

41. Alkilany, A. M.; Thompson, L. B.; Boulos, S. P.; Sisco, P.N.; Murphy, C. J.; Adv. Drug. Deliv. Rev. 2012, 64, 190.

42. Abbas, A.; Lichtman, A.; Pillai, S.; Cellular and molecular immunology, $8^{\text {th }}$ ed., Elsevier: Philadelphia, 2014.

43. Boulos, S. P.; Davis, T. A.; Yang, J. A.; Lohse, S. E.; Alkilany, A. M.; Holland, L. A.; Murphy, C. J.; Langmuir 2013, 29, 14984.

44. Vonnemann, J.; Beziere, N.; Böttcher, C.; Riese, S.B.; Kuehne, C.; Dernedde, J.; Licha, K.; von Schacky, C.; Kosanke, Y.; Kimm, M.; Meier, R.; Ntziachristos, V.; Haag, R.; Theranostics 2014, 4, 629.

45. Zhu, H.; Chen, Y.; Yan, F-J.; Chen, J.; Tao, X-F.; Ling, J.; Yang, B.; He, Q-J.; Mao, Z-W.; Acta Biomaterialia 2016, doi: 10.1016/j. actbio.2016.12.050.

46. Hunter, R. In Zeta potential in colloid science: principles and applications; Ottewilla, R. H., Rowell, R. L., eds.; Academic Press: London, 1981.

47. Chumakova, O. V.; Liopo, A. V.; Andreev, V. G.; Cicenaite, I.; Evers, B. M.; Chakrabarty, S.; Pappas, T. C.; Esenaliev, R. O.; Cancer Lett. 2008, $261,215$.

48. Liopo, A.; Conjusteau, A.; Tsyboulski, D.; Ermolinsky, B.; Kazansky, A.; Oraevsky, A.; J. Nanomed. Nanotechnol. 2012, S2, 1. 